\title{
Study on deep learning political culture communication system in Universities under the perspective of postmodern media
}

\author{
Wenjuan Zhu ${ }^{1}$, Feng Wang ${ }^{2,}$, Xianping Yu ${ }^{1}$, Yibin Huang ${ }^{1}$, Huajie Sui ${ }^{3}$ \\ ${ }^{1}$ School of Pharmacy, Jiangxi University of Traditional Chinese Medicine, Jiangxi, 330004, China \\ ${ }^{2}$ School of Computing, Jiangxi University of Traditional Chinese Medicine, Jiangxi, 330004, China \\ ${ }^{3}$ School of Humanities, Jiangxi University of Traditional Chinese Medicine, Jiangxi, 330004, China \\ *Corresponding author
}

Keywords: Artificial intelligence, modern media, ideological and political education, deep learning, neural network.

\begin{abstract}
With the development of industrialization and computer science and technology, people pay more and more attention to the intelligent industry, big data applications in industry by leaps and bounds, artificial intelligence in various fields show their unique style. In this paper after the modern media from the perspective of Ideological and political culture to establish research study of artificial intelligence network based on the depth of political culture in universities, construct the perspective of postmodern media dissemination system. This paper is based on the MATLAB platform, constructing artificial intelligence after modern media from the perspective of political culture communication system based on its operation is simple, does not require advanced programming background. Based on probabilistic neural network, the colleges and universities from the perspective of modern media thought political and cultural communication system construction, and validation is based on probabilistic neural network artificial intelligence forecasting has good convergence and the ability of fault tolerance and data processing ability.
\end{abstract}

\section{Introduction}

From the perspective of postmodern media is an ideological foundation based on post modernism through modern media angle to reflect the. After the twentieth Century, after the whole world to set off a sense of extension, not only has a modern criticism of some of the drawbacks of the phenomenon, colleagues have their own criticism of the color has two sides. Is the current ideological and political education in Colleges and universities need to strengthen attention and prevention of. In the now highly developed media today, modern media perspective more and more by the more attention, especially in the aspect of college students and ideological and political construction. After the modern way of thinking is the one hand for college students to provide a new way of thinking and a broader perspective, is conducive to better college students to improve their own treatment of the problem of comprehensive thinking and attention. But on the other hand, it is easy to make the criticism and suspicion of the correct values of college students, and it is the important aspect of the ideological and political culture construction in Colleges and universities. Therefore, it is of great practical significance to construct the ideological and political culture of the University from the perspective of modern media, which can provide a reliable prediction and learning model for the ideological and political culture construction in Colleges and universities.

After the second industrial revolution, a large number of machines used to promote the development of human civilization, and human medical diagnosis similar system simulation development process into three stages: the first stage is the primitive stage, just a simple processing. The second stage is the development of testing technology and sensing technology, mainly processing signal. The third stage is the stage of intelligent simulation technology, processing and forecasting data, making all kinds of system simulation with [1] rapid development. In recent 
decades, people in the mining industry potential bionic algorithm, in many research fields showing broad application prospects. In the field of industrial use oriented object programming language and database for real-time tracking control, for teaching and research, master the complex language is not conducive to the students to cultivate an interest, this paper based on MATLAB for the construction of expert system, for the students and teachers in Colleges and universities, do not need to program design of complex and advanced, and only need to call the function and simple programming can be. For the University of modern media from the perspective of political culture and provide reference to spread simulation.

\section{Model}

After Colleges and universities from the perspective of modern media ideological and political and cultural communication systems depth study, to neural network based learning system, with the quantization of the data, so as to achieve understanding of rational quantification of belief system, social relations, modern rationality, knowledge system, value concept, through neural network learning and training. The next step is to determine the results according to the information processing to predict the development trend and puts forward corresponding methods ${ }^{\text {[2] }}$. Including the dynamic adjustment of College Ideological and political teaching methods appropriate reform, effects of demonstration role for the reasonable application of the network media, students and exemplary role to play, right of college campus culture construction of harmonious etc. measures.

$\mathrm{BP}$ neural network is a kind of bionic algorithm, which has two main features. The first feature is the transfer of information, and the second feature is the error back propagation. No interactions between the neurons and change the value with genetic effect, repeatedly until the desired error, the training in line with the expected proportion range matrix ${ }^{[3]}$.

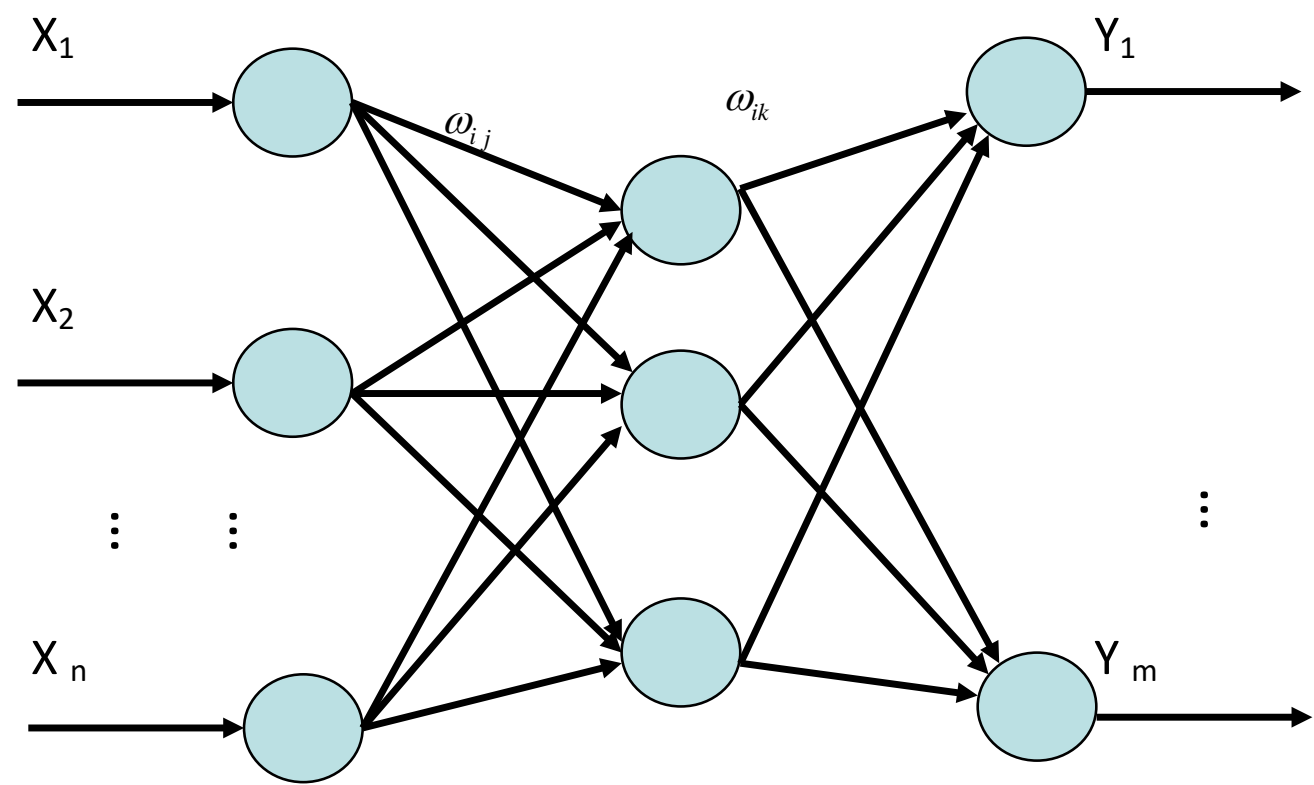

Fig 2-1 structure of the anterior neural network

Figure 2-1, the $\mathrm{X}_{1}, X_{2}, \cdots X_{n}$ input values for the forward propagation, the $\mathrm{Y}_{1}, Y_{2} \cdots Y_{\mathrm{m}}$ predicted value for the reverse propagation, $\omega_{i j}$ and the $\omega_{\mathrm{jk}}$ proportion of the reverse propagation.

In Figure 2-1, back propagation neural network is a nonlinear function, independent variables as network input value, the dependent variable is the network output value, which can construct a from $\mathrm{n}$ to $\mathrm{m}$ dimension function.

The training network can make the data become standard, the network is more intelligent. The training steps are as follows:

Step 1: initialize the network. According to type, $\operatorname{print}(\mathrm{X}, \mathrm{Y})$ matrix algorithm to determine the number of nodes $\mathrm{n}$, the number of hidden layer nodes $l$, the number of print layer nodes $m$. And 
initialize type layer $\omega_{i j}$ and printing layer $\omega_{\mathrm{jk}}$ neurons between the connection and the proportion a , invisible layer range $b$, the print range and given access rate and agitation function.

The second step: the hidden layer output. According to the matrix X, $Y$ to determine the type of node numbers $\mathrm{n}$, contact connection between the layers $\omega_{i j}$ and $a$ range to calculate the proportion of hidden layer output $H$.

$$
H_{j}=f\left(\sum_{i=1}^{n} \omega_{i j} x_{i}-a_{j}\right) \quad j=1,2, \cdots, l
$$

Type 2-1, $\quad$ is hidden layer nodes; $f$ agitation function.

The third step: print output. The contact layer connection proportion $\omega_{\mathrm{jk}}$ and range output $\mathrm{H}, \mathrm{b}$ evolutionary computation algorithm to predict the output $O$ 。.

$$
O_{k}=\sum_{1}^{l} H_{j} \omega_{j k}-b_{k} k=1,2, \cdots, m \quad 2-2
$$

Fourth step: error calculation. According to the prediction of the print $O$ and expected to print $Y$, calculate the prediction error e.

$$
e_{k}=Y_{k}-O_{k} k=1,2, \cdots, m
$$

The fifth step: the proportion of updates. Based on the $e$ prediction error updating algorithm $w_{i j}$, $w_{j k} \cdot$

$$
w_{i j}=w_{i j}+\eta H_{j}\left(1-H_{j}\right) x(i) \sum_{k=1}^{m} w_{j k} e_{k} j=1,2, \cdots, n j=1,2, \cdots, l \quad 2-4
$$

Type 2-4, $\eta$ for learning rate.

Step sixth: update range. According to the prediction errore update range $a, b$.

$$
\mathrm{a}_{\mathrm{j}}=a_{j}+\eta H_{j}\left(1-H_{j}\right) \sum_{k=1}^{m} w_{j k} e_{k} j=1,2, \cdots, l b_{k}=b_{k}+e_{k} \quad k=1,2, \cdots l \quad 2-5
$$

The seventh step: to determine whether the end, if not up to the standard, return steps $2^{[4]}$.

Neural network was first proposed by D.F.Specht in 1989 .This kind of neural network is a kind of parallel, based on probability theory. This algorithm has many advantages, one is the classification ability and multi-dimensional processing ability. And the prediction accuracy is high.

The PNN network is a feed forward type bionic algorithm, and its theory is based on the Bayesian minimum risk criteria. The algorithm developed from radial basis, it is very suitable for pattern recognition ${ }^{[5]}$.

The algorithm model of this paper is composed of four layers, which are: type layer, model layer, weighted layer, print layer, and so on. Its basic structure is shown in figure 2-2. 


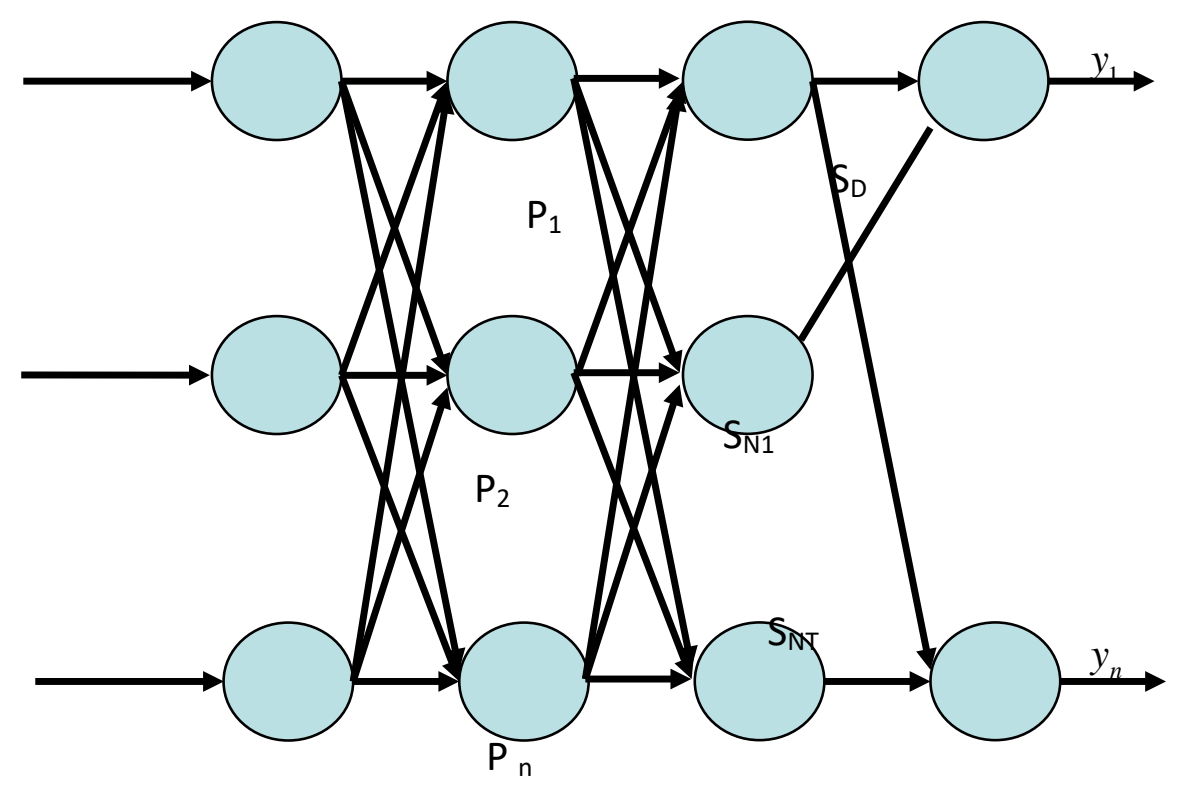

Figure 2-2 basic structure diagram

Type a value to be converted into a feature vector to the next layer of network, the sample dimension is the number of neurons, and the number of training samples of each class is equal to the number of neurons in the model layer. The output of each unit of the model layer is:

$$
\mathrm{f}(X, W)=\exp \left[\frac{\left(X-W_{i}\right)^{T}\left(\mathrm{X}-\mathrm{W}_{\mathrm{i}}\right)}{2 \delta^{2}}\right] \quad 2-6
$$

Type in the $W_{i}$ proportion of the type layer to the model layer. Smoothing index $\delta$.

The weighted layer is the statistical value, calculated by the formula PDF, get 2-6. The weighted layer is distributed according to the category, and the other units are not connected by class. The greater the probability estimates, the more the weighted layer output, the print layer and then do the normalization processing.

PNN exclusion prediction algorithm is described as follows: two models are known to assume: $\theta_{\mathrm{A}} 、 \theta_{\mathrm{B}}$. For distinguishing feature samples $\mathrm{X}=\left(\mathrm{x}_{1}, \mathrm{x}_{2}, \cdots \mathrm{x}_{\mathrm{n}}\right)$;

$$
\begin{aligned}
& \text { If } h_{A} l_{A} f_{A}>h_{B} l_{B} f_{B} \text {, then } X \in \theta_{\mathrm{A}} . \\
& \text { If } h_{A} l_{A} f_{A}>h_{B} l_{B} f_{B} \text {, then } X \in \theta_{\mathrm{A}} .
\end{aligned}
$$

The number of $h_{A} 、 h_{\mathrm{B}}$ training samples in the model $\theta_{\mathrm{A}} 、 \theta_{\mathrm{B}}$, the prior probability $\left(h_{\mathrm{A}}=\frac{N_{A}}{N}\right.$ 、 $\left.h_{B}=\frac{N_{B}}{N}\right) N_{A} 、 N_{B}$ and the model $N$, the $l_{A}$ total number of the training samples $\theta_{\mathrm{A}}$. The penalty factor. The so-called penalty factor, $\mathrm{X}$ is to belong to the sample error $\theta_{\mathrm{B}}$ of the points into $\theta_{\backslash \mathrm{A}}$; for the model $f_{A} 、 f_{B}$, the $\theta_{\mathrm{A}} 、 \theta_{\mathrm{B}}$ probability density function (Probability, Density Function PDF).

An estimate method is proposed by Parzen in 1962. Using this method to obtain probability approximation function. The estimated formula is as follows:

$$
f_{A}(X)=\frac{1}{(2 \pi)^{\frac{P}{2}} \delta^{P}} \frac{1}{m} \sum \exp \left[-\frac{\left(X-X_{a i}\right)^{T}\left(X-X_{a i}\right)}{2 \delta^{2}}\right]
$$

The first training $i$ vector in the formula $X_{a i}-\theta_{\mathrm{A}}$.

$m$-Pattern $\theta_{\mathrm{A}}$ training sample number.

$\delta$-Smoothing factor. 


\section{Solving Model}

There are some problems in neural network modeling. The selection of feature vectors must be the correct reflection of the characteristics of the problem. It is important to include enough information to be identified in the feature. In neural network establish learning system, add the quantization of data processing, so as to achieve understanding of rational quantification of belief system, social relations, modern rationality, knowledge system, value concept, through neural network learning and training. The next step is to predict the development trend based on the results of the information to predict its development trend and then put forward the corresponding countermeasures. The input feature vector of the bionic algorithm is the belief system, the social relation, the modern reason, the knowledge system, the value idea. Learning degree judgment type for output matrix ${ }^{[6] .}$

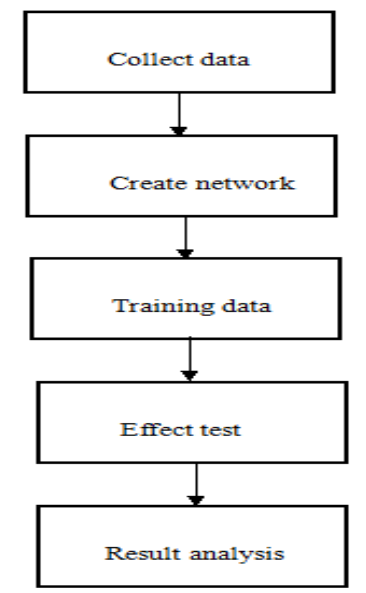

Figure 3-1 algorithm steps design flow chart

Collected data for $33 \times 4$ dimensional matrix. Before selecting a number of (such as 23) out of the sample, as a network training samples, selected after a number of (such as 8) samples as a validation sample. The input layer of neural network is three ratio method, and the output is the result of the system simulation and identification. The code of the simulation type corresponds to the table 3-1.

Table 3-1 simulation results type corresponding coding see table

\begin{tabular}{cc}
\hline Prediction type & code \\
\hline Worse & 1 \\
Poor & 2 \\
commonly & 3 \\
good & 4 \\
excellent & 5 \\
\hline
\end{tabular}

After the modern media perspective of College Ideological and political culture communication system classification prediction model of the bionic algorithm to create a good, through the MATLAB to establish the two layer network algorithm. Classification layer (cluster) and competition layer, respectively. PNN network to create a direct function provided by net=newpnn MATLAB (P, T, spread). , as mentioned before, the input vector, the threshold processing is completed after the input to the function ${ }^{[7]}$.

After the data is loaded, select the type of the print matrix, and then turn the desired class into a vector. In this paper, the algorithm is designed to train the network, if the result is consistent with the use of built-in functions to predict and visualize the results. 


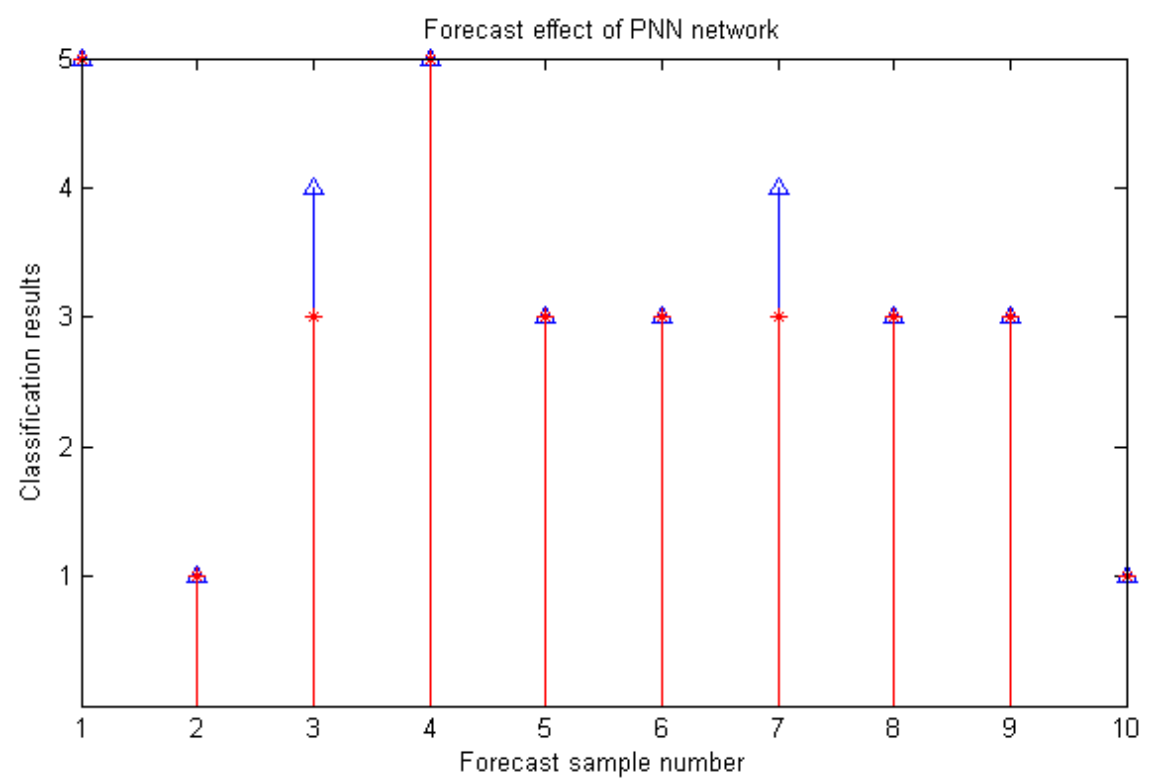

Figure 3-2 forecast results

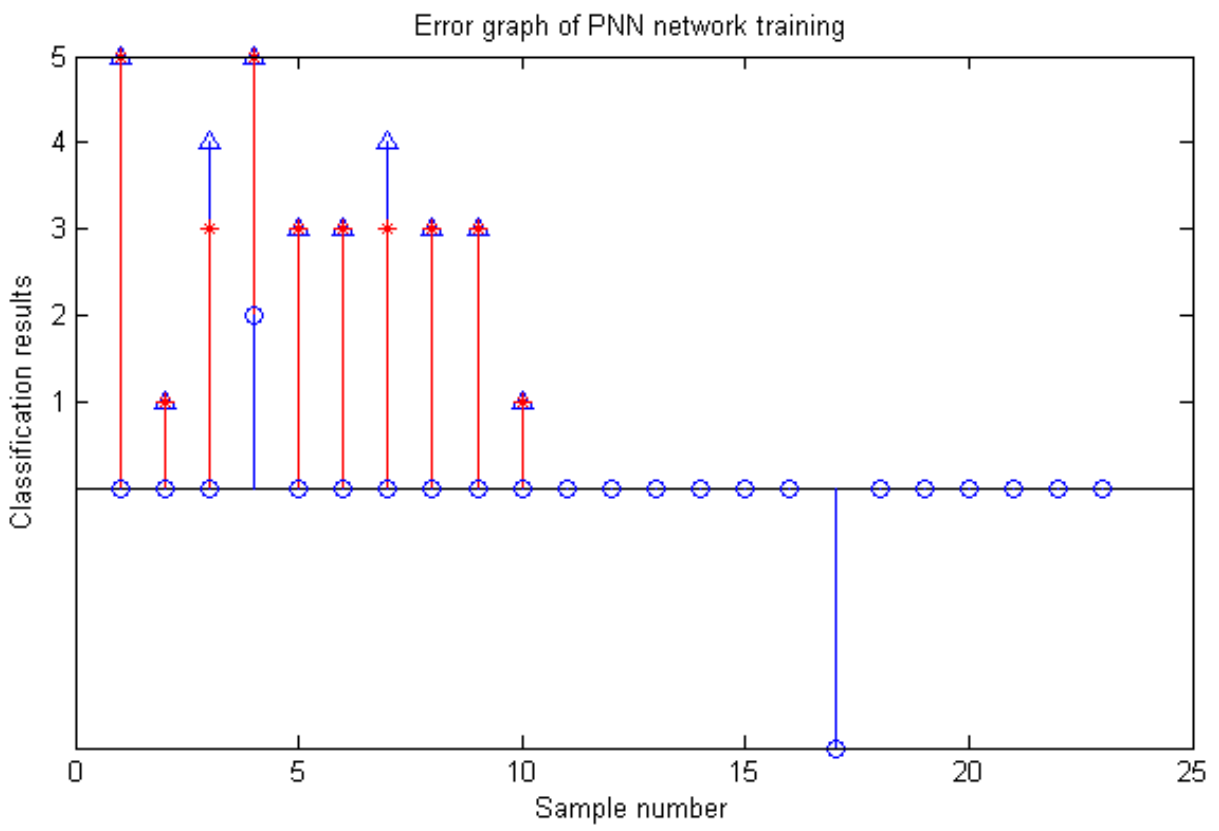

Figure 3-3 training residual diagram 


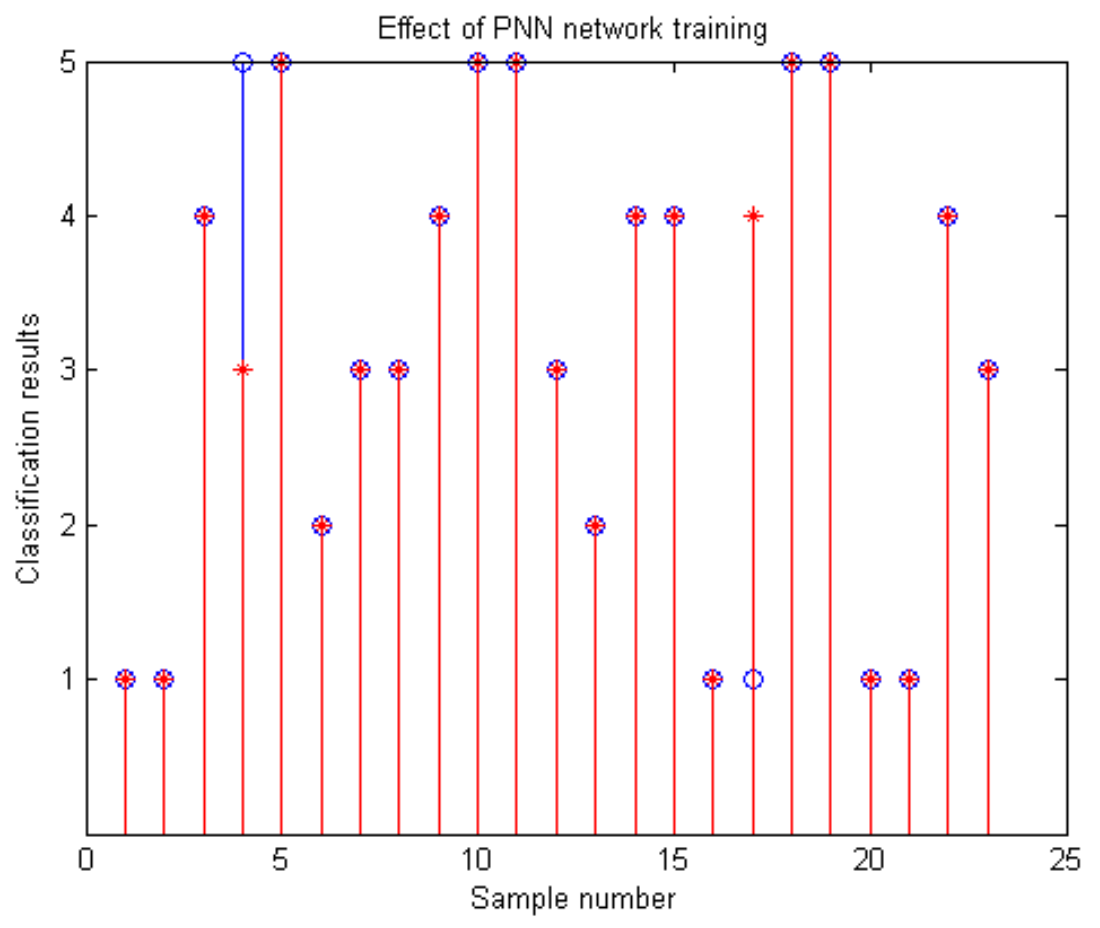

Figure 3-4 training results chart

In the depth of the intelligent bionic algorithm, there are two factors that restrict the accuracy of the algorithm. One is the number of training data, and the other is the selection of $\mathrm{S}$ value. According to the research results show that the more samples, the more extensive the type, the more accurate the results of the prediction, and the lower the spread value, the more accurate the prediction results. Through the simulation and literature studies show that for BP neural network for its rate of convergence is Pacific stability and sample additional fault-tolerant ability needs to be improved, this algorithm is robust to the above problems. The prediction results are shown in table 3-2.

Table 3-2 propagation system prediction results table

\begin{tabular}{|c|c|c|c|c|}
\hline Forecast sample number & $\begin{array}{l}\text { Forecast } \\
\text { results }\end{array}$ & classification & $\begin{array}{l}\text { Actual } \\
\text { results }\end{array}$ & classification \\
\hline 1 & & 5 & & 5 \\
\hline 2 & & 1 & & 1 \\
\hline 3 & & 4 & & 3 \\
\hline 4 & & 5 & & 5 \\
\hline 5 & & 3 & & 3 \\
\hline 6 & & 3 & & 3 \\
\hline 7 & & 4 & & 3 \\
\hline 8 & & 3 & & 3 \\
\hline 9 & & 3 & & 3 \\
\hline 10 & & 1 & & 1 \\
\hline
\end{tabular}

\section{Conclusions}

Based on the depth study of the artificial intelligence network, this paper constructs the ideological and political culture communication system of College Ideological and political culture from the perspective of post - modern media. To construct the ideological and political culture communication system from the perspective of post modern media, and to verify that the artificial intelligence based on probabilistic neural network has a good convergence and fault tolerance ability and large data processing capacity. Through the matrix experiment, we can draw the conclusion that the depth study of artificial intelligence network has several advantages: 
(1) the process model is simple and the convergence is good.

(2) the convergence effect of the network is better than that of the Bayes optimal solution, and the stability is good. There is a clear theoretical support, you can make a reasonable explanation, with convincing.

(3) sample fault tolerance capability is strong, the proportion of each other independent, increase and reduce the sample is very simple and convenient, high accuracy of prediction results, with superior judgment accuracy.

To sum up, the construction of the post modern media perspective of College Ideological and political culture communication system in terms of speed and fault tolerance performance is excellent. Therefore, it is of great practical significance to construct the ideological and political culture of the University from the perspective of modern media, which can provide a reliable prediction and learning model for the ideological and political culture construction in Colleges and universities.

\section{Reference}

[1] Du Qida, Duan Huiqiong. Ideological and political education on the path of [J]. teaching and management of post-modernism, 2010

[2] Liu Chengbo, Shang Shu song. The construction and optimization of the ecological environment system of the ideological and political education of college students [J]. Journal of Wuhan Institute of Technology, 322010

[3] edited by Yang Jiangang. Artificial neural network tutorial [M]. Zhejiang University press, 2001.

[4] Tuwei Fu, Lu Lan. From the perspective of Marx's communication practice of subjectivity in Ideological and political education theory of [J]. education, 2013, 7

[5] Xiong Jianqiu, Li Zuoyong. Using probabilistic neural network to predict the stability of hydropower slope [J]. subgrade engineering. 2006 (04).

[6] Li Xialing, Li Minlun. The relationship between the subject and object of Ideological and political education in the view of post modernism to examine the [J]. school party building and ideological education.2010 (2).

[7] Douglas Kellner, dingning. Between media culture between modern and postmodern cultural studies, identity and politics [M]. Beijing: the Commercial Press, 2013. 